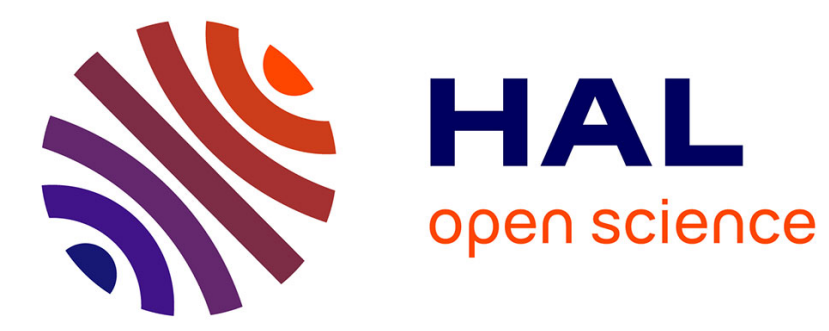

\title{
The speed of sound in periodic ducts
}

Serena Russo, David Fabre, Flavio Giannetti, Paolo Luchini

\section{To cite this version:}

Serena Russo, David Fabre, Flavio Giannetti, Paolo Luchini. The speed of sound in periodic ducts. Journal of Sound and Vibration, 2016, 361, pp.243-250. 10.1016/j.jsv.2015.09.045 . hal-01337524

\section{HAL Id: hal-01337524 https://hal.science/hal-01337524}

Submitted on 7 Jul 2016

HAL is a multi-disciplinary open access archive for the deposit and dissemination of scientific research documents, whether they are published or not. The documents may come from teaching and research institutions in France or abroad, or from public or private research centers.
L'archive ouverte pluridisciplinaire HAL, est destinée au dépôt et à la diffusion de documents scientifiques de niveau recherche, publiés ou non, émanant des établissements d'enseignement et de recherche français ou étrangers, des laboratoires publics ou privés. 


\section{OATAO \\ Open Archive Toulouse Archive Ouverte}

\section{Open Archive TOULOUSE Archive Ouverte (OATAO)}

OATAO is an open access repository that collects the work of Toulouse researchers and makes it freely available over the web where possible.

This is an author-deposited version published in : http://oatao.univ-toulouse.fr/ Eprints ID : 15887

To link to this article : DOI:10.1016/j.jsv.2015.09.045

URL : http://dx.doi.org/10.1016/j.jsv.2015.09.045

To cite this version : Russo, Serena and Fabre, David and Giannetti, Flavio and Luchini, Paolo The speed of sound in periodic ducts.

(2016) Journal of Sound and Vibration, vol. 361. pp. 243-250. ISSN 0022-460X

Any correspondence concerning this service should be sent to the repository administrator: staff-oatao@listes-diff.inp-toulouse.fr 


\title{
The speed of sound in periodic ducts
}

\author{
Serena Russo ${ }^{\mathrm{a}, *}$, David Fabre ${ }^{\mathrm{b}, \mathrm{c}}$, Flavio Giannetti ${ }^{\mathrm{a}}$, Paolo Luchini ${ }^{\mathrm{a}}$ \\ a Universita' degli Studi di Salerno, Italy \\ ${ }^{\mathrm{b}}$ Université de Toulouse, IMFT (Institut de Mécanique des Fluides de Toulouse), France \\ ${ }^{\mathrm{c}}$ University of Toulouse, France
}

\begin{abstract}
A B S T R A C T
The flow through corrugated pipes is known to lead to strong whistling tones which may be harmful in many industrial appliances. The mechanism is known to originate from a coupling between vortex shedding at the edges of the cavities forming the wall of the tube and the acoustical modes of the pipe. The latter depend upon the effective velocity of sound $c_{\text {eff }}$ within the corrugated pipe. The purpose of this paper is to compute accurately this effective velocity of sound through an asymptotic calculation valid in the long-wave limit. Results are given for a number of geometries used in previous works, and compared with a simple model in which the effective speed of sound is function of the geometry of the pipe. The latter is found to work best for short cavities but significant disagreement is found for longer cavities. The case of $2 \mathrm{D}$ channels with a corrugated wall is also considered.
\end{abstract}

\section{Introduction}

Corrugated pipes are commonly used in many engineering and industrial applications because they combine local rigidity with global flexibility. In many cases, the flow of air through such pipes can cause a loud whistling at specific frequencies. In most industrial cases, such whistling is an undesired effect as it may result in severe noise and structural vibration problems (see [1] for a recent review, covering industrial applications and available work on this problem). On the other hand, the ability of such ducts to generate clear and tonal sound is also used in a musical toy called the "hummer" or "Voice of the dragon" [2].

The mechanism responsible for whistling is known to combine an hydrodynamic and an acoustic subsystem [3,4]. The shear-layer instability, which is the hydrodynamic subsystem, acts as an amplifier and supplies acoustic energy to the system. On the other hand, the acoustic subsystem is represented by longitudinal standing waves, which act as a band-pass filter and maintain the synchronization in this feedback mechanism [5]. Note that the mechanism shares some similarity with the resonant cavity mechanism studied by Rossiter [6] for transonic flows except that the frequency is selected by the whole length of the tube, not the size of a single cavity. In this paper, only the acoustic part of the system is taken into account, then no mean flow is considered.

In a straight smooth tube of length $L^{* 1}$ opened at both sides (where the definition of $L^{*}$ may contain end corrections to account for matching with the outer regions), the acoustical frequencies are classically given by $f_{m}^{*}=m f_{1}^{*}=\frac{m c_{0}^{*}}{2 L^{*}}$ $(m=1,2,3, \ldots)$ where $c_{0}^{*}$ is the speed of sound in unbounded medium. In the present case of a corrugated tube with periodic

\footnotetext{
* Corresponding author.

${ }^{1}$ In this paper all the dimensional quantities are indicated with the asterisk.
} 
geometry, the presence of cavities at the walls results in an effective speed of sound $c_{\text {eff }}^{*}$ which is lower than that in unbounded space. In the case where the mean flow corresponds to a low Mach number (which is effectively the case in most applications), the resonance frequencies are given by

$$
f_{m}^{*}=m f_{1}^{*}=\frac{m c_{\mathrm{eff}}^{*}}{2 L^{*}}, \quad m=1,2,3, \ldots
$$

so the knowledge of the effective speed of sound $c_{\text {eff }}^{*}$ is thus crucial to predict the whistling frequencies of such devices.

Research on acoustic propagation has encompassed waveguides having various cross-sectional geometries, wall impedances and steady flow fields. For two-dimensional ducts, straightforward expansions of the form $\phi_{0}+\epsilon \phi_{1}+\cdots$ were obtained by Isakovitch [7] for the case of a waveguide with only one sinusoidally undulating wall, by Samuels [8] for the case of a waveguide with in-phase wall undulations, and by Salant [9] for the general problem. In particular, in [9], he considers the propagation of acoustic waves through a plane waveguide consisting of two rigid sinusoidal walls by using a multiple scale approach. The same approach can be found in [10-12]. Nayfeh [10] discusses sound waves in twodimensional ducts with sinusoidal walls. In [11], the same author extends this analysis to the case of linear waves propagating in a cylindrical hard walled duct whose wall has weak undulations which need not to be periodic. In [12], Nusayr uses the same method of multiple scales to analyze wave propagation in a rectangular hard-walled duct whose walls have weak periodic undulations.

Methods using a different approach have been suggested in the literature. In particular, in [13], Pagneux et al. present a multimodal expansion [14] to the problem of determining the wave propagation in waveguides with varying cross section. The same approach is used in $[15,16]$, where a multimodal method is used to analyze the wave propagation in waveguides containing restrictions (or corrugations) with circular arc shapes. When the geometry of the duct is complex or it is not possible to derive a geometrical transformation to a simpler configuration, this approach may pose numerical difficulties in solving the equations, unlike the method of multiple scales. The propagation of time-harmonic acoustic waves in a cylindrical hard-walled duct has been also studied with the null field approach based on Green identities (see [17,18]).

Unlike what is done in [10-12], where the multiple scale approach is applied to geometries characterized by weak undulations of the wall whose amplitude is small compared with the height of the channel; here, there are no restrictions about the amplitude of the wall because the present formulation relies on the assumption that the acoustical wavelength is large compared with the characteristic dimension of the single corrugation, which is verified in most applications. Moreover, despite the multimodal method, it can be also applied to very complex configurations without consider any geometrical transformation.

The results obtained with our approach will be compared with a simple model, proposed by Cummings and initially published by Elliott [19], that gives an estimation of the effective speed of sound as a function of the geometry of the duct

$$
\left(\frac{c_{\text {eff }}^{*}}{c_{0}^{*}}\right)^{2}=\frac{V_{\text {in }}}{V_{\text {tot }}}
$$

where $V_{\text {in }}$ is the volume of the inner part of the duct (excluding the cavities) and $V_{\text {tot }}$ is the total volume (including the cavities). The argument leading to Eq. (2) relies on a quasi 1D modeling of Euler equations in which it is assumed that the velocity fluctuations do not vary across the pipe cross-section and the corrugations are modeled as small perturbation to the radial velocity. The mathematical derivation leading to Eq. (2) can also be found in [1], and a simpler derivation (in the case where the mean flow is neglected) is reproduced in the appendix of the present paper.

In Section 2, we detail the mathematical analysis and the numerical procedure. Then in Section 3 we provide results for a variety of geometries of tubes used in the literature, and compare with the simple estimate of Cummings-Elliott. Our analysis also works for 2D channels, and we briefly consider such geometries in Section 4. Then Section 5 summarizes the results and the open issues.

\section{Mathematical analysis and numerical approach}

The problem considered here is the propagation of acoustic waves in a corrugated pipe, which is assumed to be a periodic domain in the axial $(x)$ direction. Fig. 1 depicts the typical geometry considered in the sequel: this description holds for both axisymmetric pipes (the lower boundary corresponding to the symmetry axis) and for two-dimensional channels (the lower boundary corresponding to a wall).

Let us consider an infinite pipe having a geometry like that shown in Fig. 1 as a fundamental building block. The numerical domain corresponds to a single periodic cell of pitch $P_{t}^{*}$ of the corrugated pipe so the solution is imposed to be periodic along the length of the pipe. We describe the acoustic field through the velocity potential $\Phi(x, r)$, nondimensionalized with the characteristic length of the pipe $L^{*}$ and $c_{0}^{*}$, where $x=x^{*} / L^{*}$ and $r=r^{*} / L^{*}$ are respectively the streamwise and radial coordinates. 


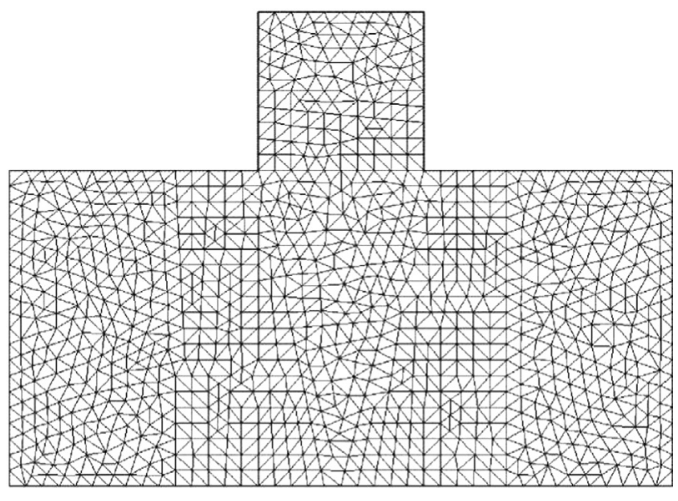

Fig. 1. Sample mesh for the elementary computational domain.

In the hypothesis in which there is no mean flow, the latter obeys the Helmholtz equation in the domain $\Omega$, with Neumann conditions on the wall $\Gamma_{w}$

$$
\begin{gathered}
\Delta \Phi=\frac{\partial^{2} \Phi}{\partial t^{2}} \\
\text { n. } \nabla \Phi(x, r)=0,
\end{gathered}
$$

where $\mathbf{n}$ is the outward normal vector. Here we will seek a solution under Floquet form, namely

$$
\Phi(x, r)=\phi(x, r) \exp \left(i k\left[x-c_{\mathrm{eff}} t\right]\right)
$$

where $c_{\text {eff }}=\frac{c_{\text {ffr }}^{*}}{c_{0}^{*}}$, the first factor $\phi(x, r)$ is a periodic function with periodicity equal to the cell pitch, and the second factor is a modulation wave (here a progressive wave, but a standing wave could work as well). The term $c_{\text {eff }}$ is the effective speed of sound to be determined and $k=k^{*} L^{*}$. Introducing this ansatz into Eq. (3) leads to the following problem:

$$
\left[\Delta \phi+2 i k \frac{\partial \phi}{\partial x}-k^{2} \phi\right]=-c_{\mathrm{eff}}^{2} k^{2} \phi
$$

with boundary conditions

$$
\left(\mathbf{n} \cdot \nabla \phi(x, r)+i k n_{x} \phi\right)=0 \quad \text { on } \Gamma_{w}
$$

where $n_{x}$ is the projection of $\mathbf{n}$ along the axial direction.

Here we will not attempt to solve this problem in the general case, but will only consider the case where the wavelength $2 \pi / k$ is much larger than 1 , namely in the limit $k \ll 1$. We thus expand the periodic component $\phi(x, r)$ as a series in term of $k$

$$
\phi(x, r)=\phi_{0}(x, r)+i k \phi_{1}(x, r)+k^{2} \phi_{2}(x, r)+\cdots
$$

Injecting this into the problem (Eqs. (5) and (6)) and considering the successive orders in the development leads to the following linear problems which have to be solved in a domain corresponding to an elementary cell

$$
\begin{gathered}
\Delta \phi_{0}=0 ; \quad \mathbf{n} \cdot \nabla \phi_{0}=0 \quad \text { on } \Gamma_{w} \\
\Delta \phi_{1}=-2 \frac{\partial \phi_{0}}{\partial x}, \quad \mathbf{n} \cdot \nabla \phi_{1}+n_{x} \phi_{0}=0 \quad \text { on } \Gamma_{w} \\
\Delta \phi_{2}=2 \frac{\partial \phi_{1}}{\partial x}+\phi_{0}-c_{\text {eff }}^{2} \phi_{0}, \quad \mathbf{n} \cdot \nabla \phi_{2}-n_{x} \phi_{1}=0 \quad \text { on } \Gamma_{w}
\end{gathered}
$$

Mathematically, all these elementary problems are inhomogeneous Poisson problems with Neumann boundary conditions, with the following generic form:

$$
\Delta \tilde{\phi}=f ; \quad \mathbf{n} \cdot \nabla \tilde{\phi}=g \quad \text { on } \Gamma_{w}
$$

It is well known that with this kind of boundary conditions, such problems are ill-posed, and their solvability depends on a compatibility condition, which reads

$$
\int_{\Omega} f \mathrm{~d} V+\int_{\Gamma_{\mathrm{w}}} g \mathrm{~d} S=0 .
$$

If such a condition is fulfilled, the problem is solvable and the solution is defined up to an undetermined constant. On the other hand, if the condition is not fulfilled, then no solution can be found. We will thus inspect successively the solvability of these problems: 
- The first problem (Eq. (8)) defining $\phi_{0}$, is trivial, and the solutions are constant functions. For simplicity we choose $\phi_{0}=1$.

- For the second problem (Eq. (9)) defining $\phi_{1}$, the compatibility condition is easily verified, so the solution is well-defined.

- For the third problem (Eq. (10)) governing $\phi_{2}$, the compatibility condition directly leads to the following expression, which

$$
c_{\mathrm{eff}}^{2}=\left(\frac{c_{\mathrm{eff}}^{*}}{c_{0}^{*}}\right)^{2}=1+\frac{2 \int_{\Omega} \frac{\partial \phi_{1}}{\partial x} \mathrm{~d} V+\int_{\Gamma_{w}} n_{x} \phi_{1} \mathrm{~d} S}{\int_{\Omega} \mathrm{d} V}
$$

So, in practice, we only need to compute $\phi_{1}$ through inversion of the problem (Eq. (9)) (with $\phi_{0}=1$ ), and the effective speed of sound is directly deduced from (Eq. (13)). The approximate solution of the Helmholtz equation proposed here is equivalent to the local solution of the Laplace equation, in the limit of $k \ll 1$, proposed by Nederveen by using an electrical analogy in [20] and, later, a numerical procedure in [21].

As we expect, formula (13) shows that $c_{\text {eff }}$ is not function of $L^{*}$ because we are in the limit of $k \ll 1$. In these conditions, it is reasonable to consider only a single elementary cell of the pipe whose characteristic dimensions $\left(P_{t}^{*}, R^{*}\right.$, ecc.) are small compared with the total length of the pipe. For these reasons, all the results presented in the following sections are derived by using the radius of the pipe $\left(R^{*}\right)$ as reference length.

Here, $\phi_{1}$ is discretized using second-order (P2) finite elements over a triangular mesh of the domain $\Omega$ corresponding to an elementary cell. The formula given above are usable for both axisymmetric geometries (Section 3, with $\phi_{1}=\phi_{1}(x, r) ; \mathrm{d} V=r \mathrm{~d} r \mathrm{~d} x ; \mathrm{d} S=r \mathrm{~d} \ell$ ) and 2-dimensional geometries (Section 4 with $\phi_{1}=\phi_{1}(x, y) ; \mathrm{d} V=\mathrm{d} x \mathrm{~d} y ; \mathrm{d} S=\mathrm{d} \ell$ ). Note that the extension to a three-dimensional domain of more general geometry, such as an array of branching pipes, would also be straightforward.

The numerical calculations are done using the Freefem ++ software. An example of numerical mesh is displayed in Fig. 1 in which a uniform mesh density is considered with average grid size $d x=0.05$. We have verified that the mesh considered here is characterized by a good spatial convergence of the computations, for a very modest computational time on current computers. By comparing the results between this mesh and another one with double number of elements, it is possible to observe that, for all the cases, the percentage difference expressed in term of $c_{\text {eff }}^{2}$ is about $10^{-5}$.

Let us consider an infinite pipe having a geometry like that of Fig. 3(a), with a radius $R=1$, a cavity depth $H=H^{*} / R^{*}$, a cavity length $W=W^{*} / R^{*}$, a plateau $L_{p}=L_{p}^{*} / R^{*}$ (which includes any rounding) and a pitch $P_{t}=L_{p}+W$. As an illustration, we represent in Fig. 2 the structure of the velocity potential for a sample axisymmetric geometry corresponding to $P_{t}=2,=0.5, R=1, H=0.5$. Plot 2 displays the structure of the term $\phi_{1}$ from our asymptotic expansion, which obviously does not respect the homogeneous Neumann conditions of the starting problem. Accordingly, the structure of the flow is most easily understood by plotting the velocity potential in primitive coordinates, i.e. $\Phi(x, r)=\Phi_{0}(x, r)+k \Phi_{1}(x, r)+\cdots$. The term at order one in this expansion, namely $\Phi_{1}(x, r)=\phi_{1}(x, r)+x$, is plotted in Fig. 2(b). The corresponding flow $\mathbf{u}_{1}=\nabla \Phi_{1}$ is perpendicular to these iso-potential lines.

\section{Results for axisymmetric corrugated pipes}

In this section we provide a parametric study of the effective speed of sound predicted by our approach as a function of the geometrical parameters, and systematically compare our predictions with the simpler approach of Cummings-Elliott (Eq. (2)).

(a)

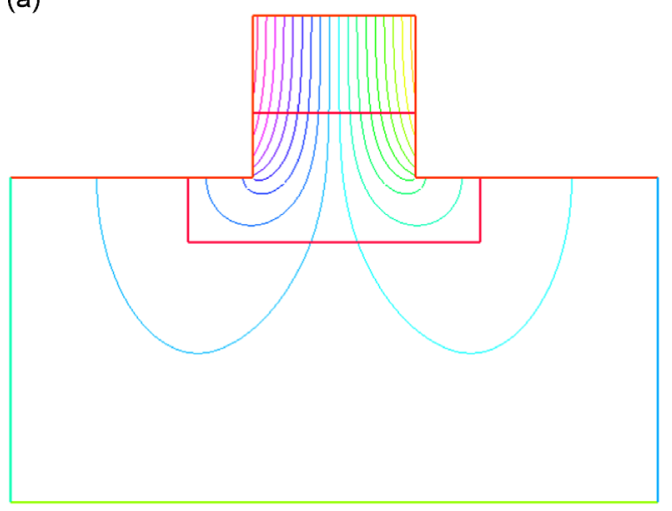

(b)

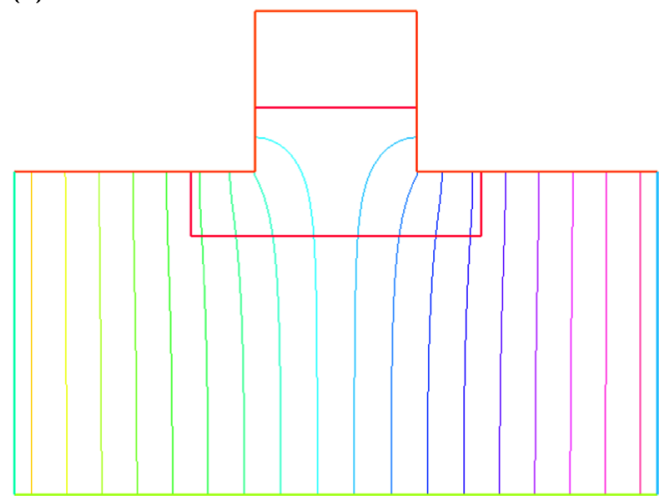

Fig. 2. Illustration of the velocity potential for a sample geometry: (a) $\phi_{1}$ and (b) $\Phi_{1}=\phi_{1}+x$. 
Table 1

Geometrical definition of investigated cases (in case B2 $r_{u p}=0.23 *$; in cases A2 and B3 $H=W$ and $P t=2 W$ ).

\begin{tabular}{|c|c|c|c|c|c|c|}
\hline Case & Ref. & Shape & $P_{t}$ & $R$ & $W$ & $H$ \\
\hline$A 1$ & [19] & Sharp & 0.4 & 1 & 0.2 & {$[0-0.5]$} \\
\hline$A 2$ & [19] & Semi-circles & {$[0-0.4]$} & 1 & {$[0-0.2]$} & {$[0-0.2]$} \\
\hline$B 1$ & [5] & Sharp & 4 & 1 & 2 & {$[0-2]$} \\
\hline$B 2$ & [5] & Sharp with upstream rounded edges $(*)$ & 4 & 1 & 2 & {$[0.3-2]$} \\
\hline$B 3$ & - & Semi-circles & {$[0.2-4]$} & 1 & {$[0.1-2]$} & {$[0.1-2]$} \\
\hline C & - & Sharp & 4 & 1 & {$[0-4]$} & 1 \\
\hline$D$ & - & Sharp & [1.1-5] & 1 & 1 & 1 \\
\hline
\end{tabular}

(a)

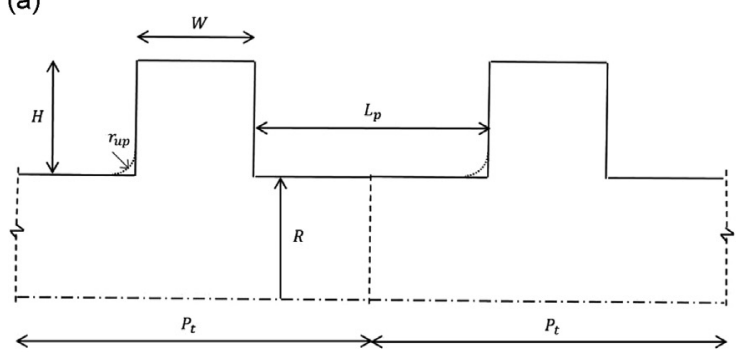

(b)

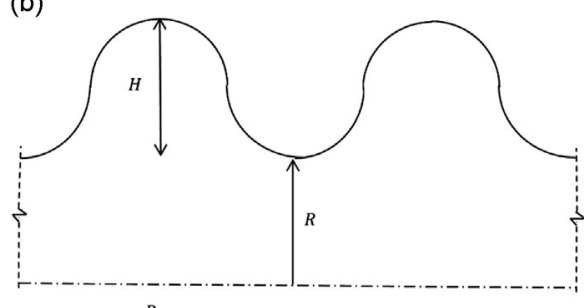

Fig. 3. Geometrical definitions for periodic pipes and channels: (a) refers to $A 1, B 1, B 2, C$ and $D$ cases and (b) refers to the $A 2$ and $B 3$ cases.

(a)

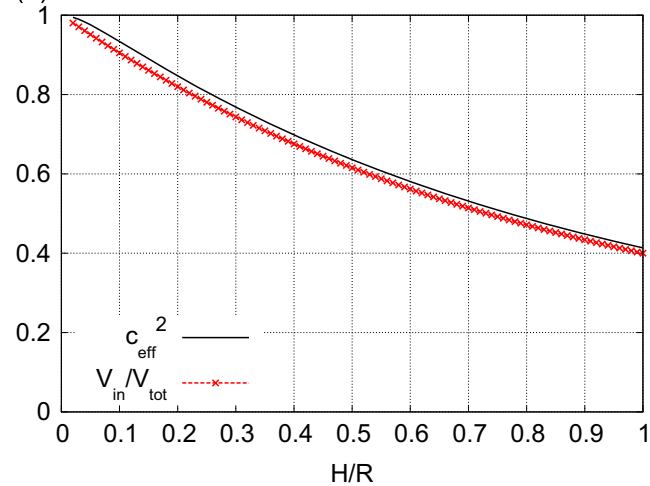

(b)

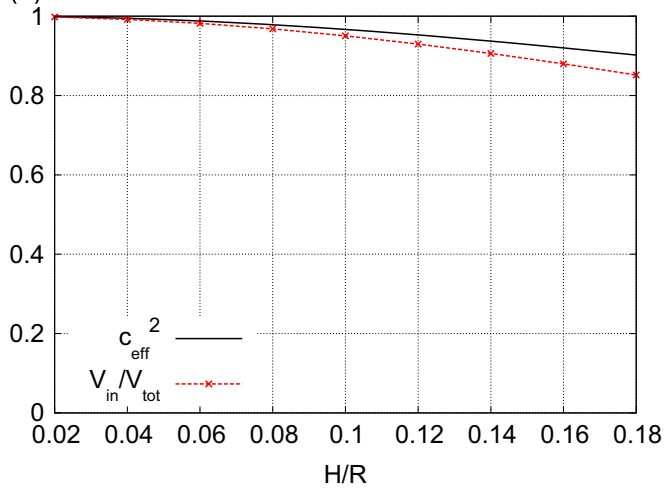

Fig. 4. Effective speed of sound vs. Cummings-Elliott approximation (Eq. (2)) as a function of cavity depth $H / R$ : (a) $A 1$ case and (b) $A 2$ case.

Table 1 describes the selected geometries. Cases A1 and B1 are chosen to study the effect of the cavity depth, with respectively a short and long pitch. Cases A2 and B2 are similar to the previous ones, but with a rounded profile (see Fig. $3 \mathrm{~b}$ for the geometric details). Finally case $C$ allows us to study the effect of the ratio $W / P_{t}$, with fixed $H$ and $P_{t}$.

Case A1 (see Fig. 4a) demonstrates that our asymptotic approach and the Cummings-Elliott approximation are in good agreement (see Fig. 4) for pipes with short cavities $(W / R=0.2$ ), even if they are quite deep (up to $H / R=1)$. In particular, the Cummings-Elliott approximation underpredicts the actual value by about 3 percent and, for $H / R>0.2$, the percentage difference between the two approaches is equal to 0.033 .

Case A2 (see Fig. 4b) shows the same trends for round cavities with $W / R<0.2$. Thus, for such short cavities, the exact shape (square or rounded) does not influence much the results, and the Cummings-Elliott approximation remains an accurate approximation of the results of our more elaborate approach.

Cases B1-B3 (see Fig. 5(a)) are similar to the previous ones, but here a longer pitch length $P_{t}$ is considered. First of all, there are no differences between the B1 and B2 cases, so the presence of the upstream rounded edge does not influence the results. Unlike what happens for the previous cases, the Cummings-Elliott approximation still captures the qualitative trends, but the disagreement is larger (about 30 percent) and is constant when $H / R>2$.

This fact is evident because the Cummings-Elliott approximation models the effect of the corrugations as small perturbations to the radial velocity, so this hypothesis fails when the ratio $H / R$ becomes very large.

Fig. 6(a) shows results for case $C$ and allows us to estimate the effect of the cavity length for a given pitch. Note that our approach predicts that the effective speed of sound behaves in a non-monotonic way as $W / R$ is increased, and reaches a minimum for $W / R \approx 2.4$. This is not surprising, as in the limit of cavity length $W$ large compared to the pipe radius $R$, the speed of sound will obviously approach the free space speed of sound $c_{0}$. This will certainly occur when increasing $W$ the 
(a)

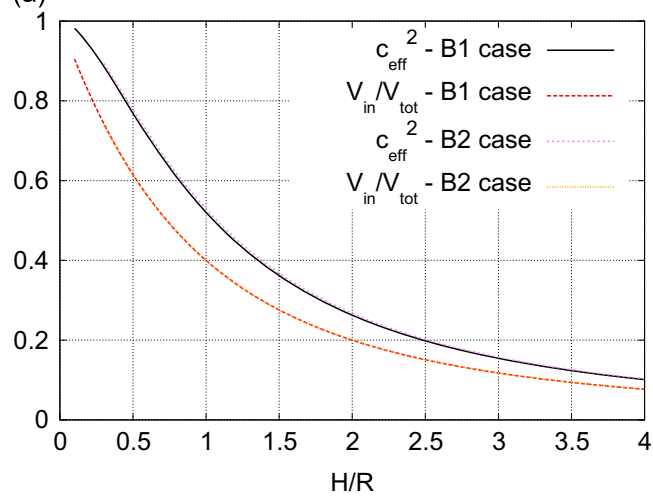

(b)

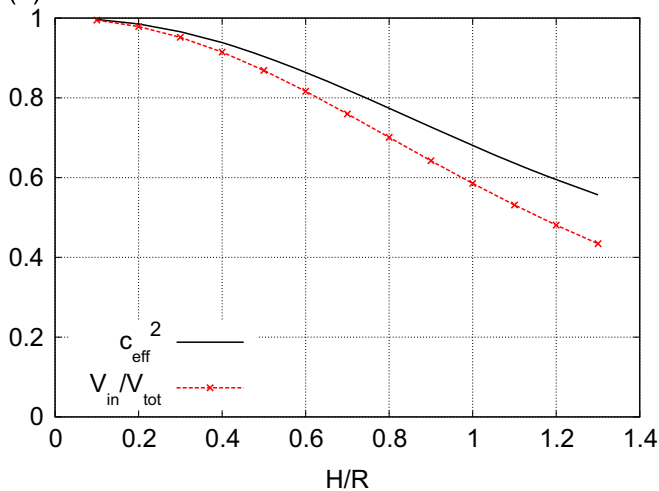

Fig. 5. Effective speed of sound vs. Cummings-Elliott approximation (Eq. (2)) as a function of cavity depth $H / R$ : (a) $B 1$ and $B 2$ cases and (b) $B 3$ case.

(a)

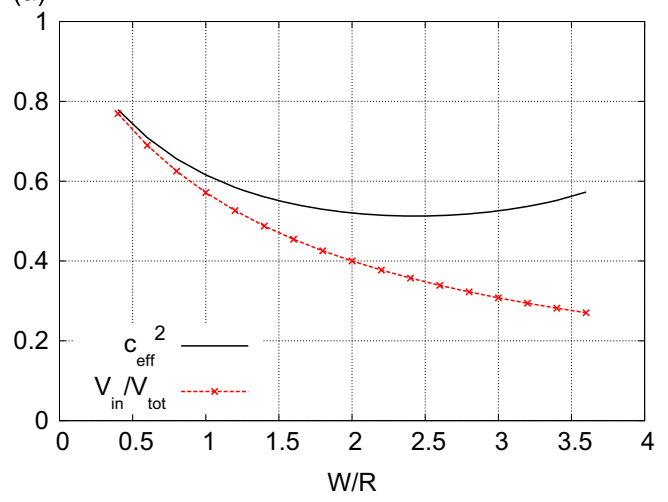

(b)

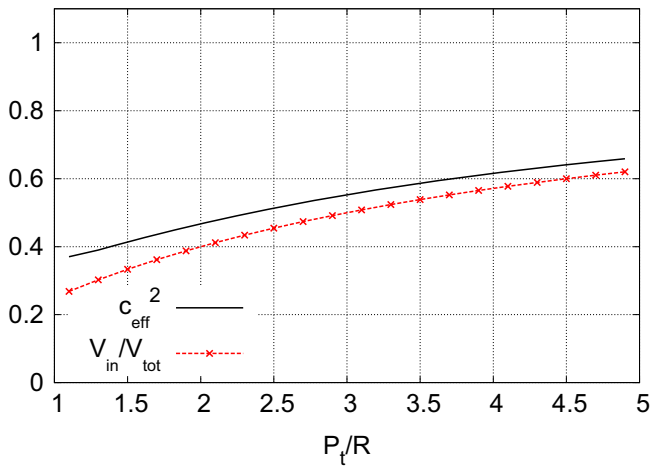

Fig. 6. Effective speed of sound vs. Cummings-Elliott approximation (Eq. (2)): (a) C case and (b) D case.

cavity becomes shallow $(W / R \gg 1)$, in fact the acoustic field penetrates deep into the cavity [21]. It is almost trivial that the approximation of Cummings, which assumes that the acoustic field does not penetrates deep into the cavity fails. Note that the approximation is quite good for $W / R<1$, which is the typical range values of $W / R$ used in corrugated tubes.

Finally case D (Fig. 6(b)) considers the effect of the variation of the spacing between the cavities, with a constant width $W=R$. Here, again, the Cummings-Elliott approximation follows the asymptotic approach, but the differences are not negligible. It can be noted that in this case, the agreement improves as the spacing between the cavities becomes larger.

\section{Results for 2D corrugated channels}

Although pipes with axisymmetric geometries are more relevant to applications, the mathematical analysis carried out in Section 2 is also directly applicable to 2D geometries (corrugated channels). In this section we provide results for such cases, using configurations similar to those analyzed in the previous section. All results are presented in Fig. 7, and are left to the reader's appreciation. The general trends, as well as the deviations with respect to the Cummings-Elliott approximation, are similar as in the case of axisymmetric pipes.

\section{Summary and discussion}

The goal of this short paper was to provide a rigorous way to compute the speed of sound in a corrugated duct, which is a key quantity for predicting the whistling frequencies, and to compare it with the simpler approximation used in most studies, due to Cummings. We provided results for a variety of geometries used in the literature, covering both axisymmetric and 2D cases. Overall, for short cavities $(W / R \leq 0.2)$, we can conclude that the results obtained by the Cummings approximation compare well with those derived by our approach, whatever the cavity depth and the exact shape of the wall (squared or rounded). On the other hand, significant departure is found for longer cavities, where the Cummings approximation significantly underpredicts the effective speed of sound. 
(a)

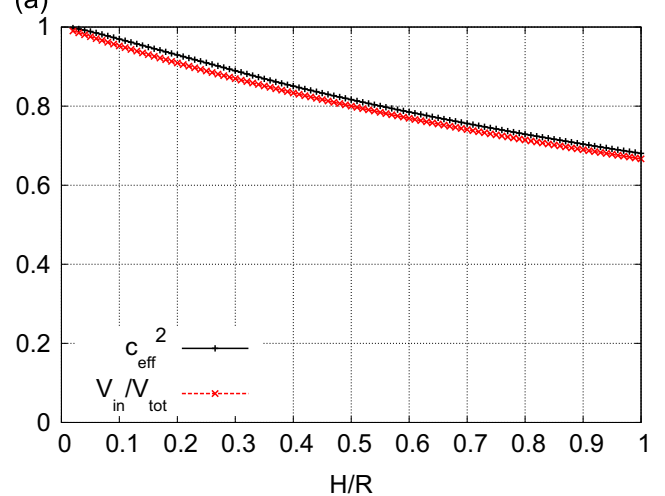

(c)

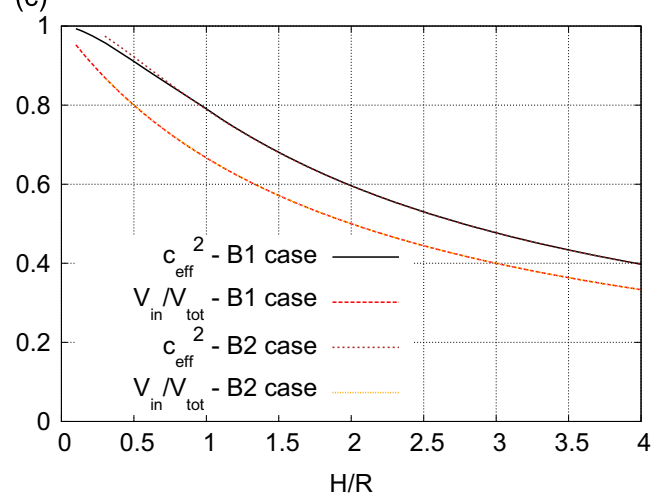

(e)

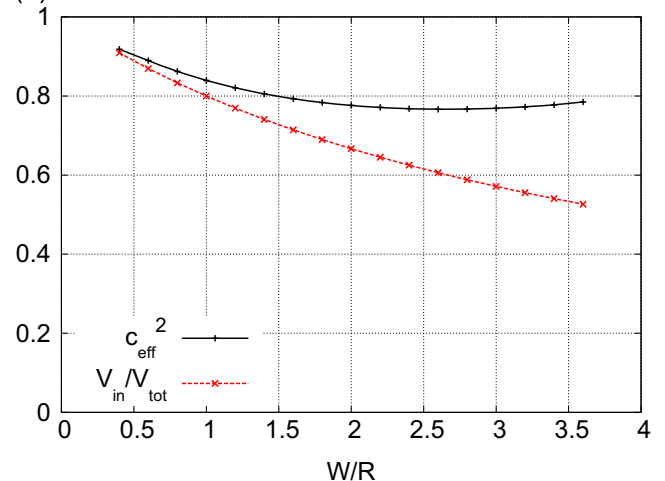

(b)

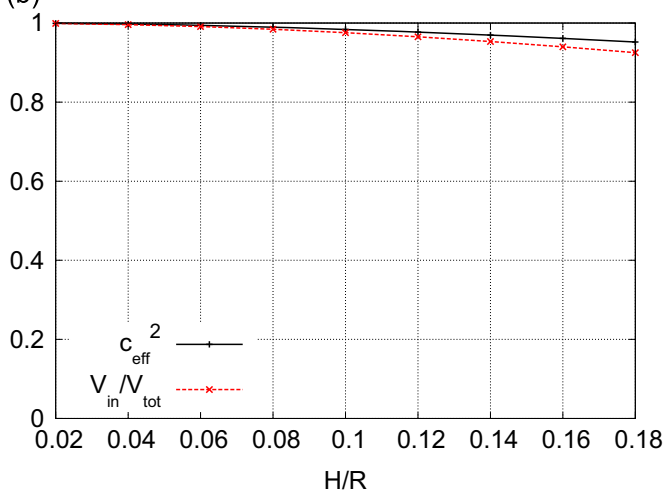

(d)

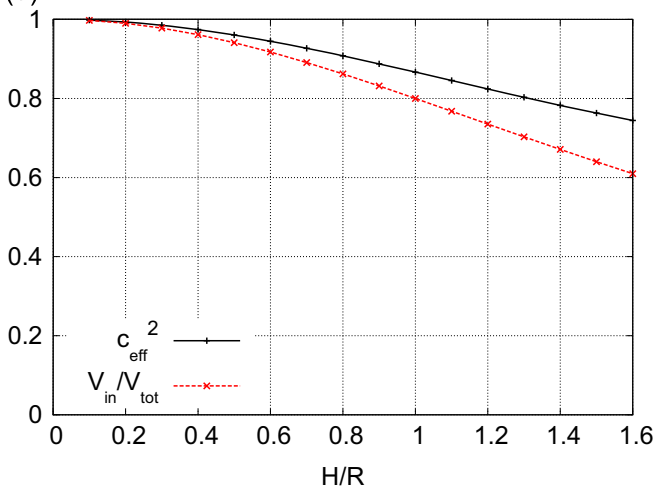

(f)

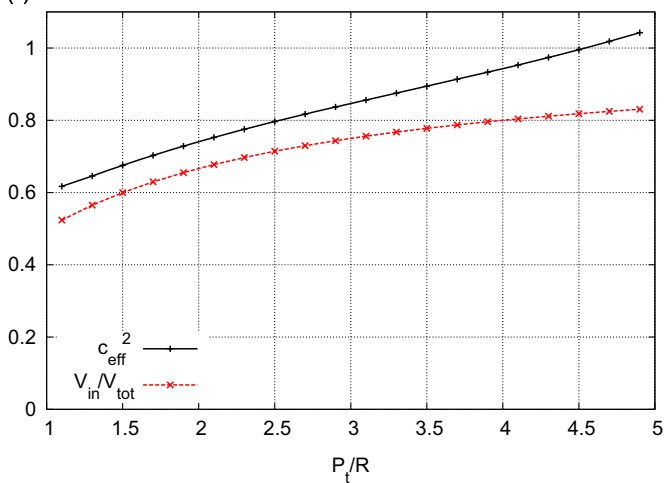

Fig. 7. Effective speed of sound vs. Cummings-Elliott approximation (Eq. (2)) for 2D geometries: (a) $A 1$ case, (b) $A 2$ case, (c) $B 1$ and $B 2$ cases, (d) $B 3$ case, (e) $C$ case, and (f) $D$ case.

Coming back to the initial problem of whistling pipes, we stress that the present approach only captures one of the two key ingredients of the phenomenon: the acoustical part responsible for frequency selection. The other key ingredient, namely vortex shedding responsible for positive energy feedback, is not addressed here, and accordingly the effect of the mean flow, and the modeling of the shear layer present in the original Cummings model, are left aside. We are currently exploring a more general treatment of the problem, including both acoustical and hydrodynamical ingredients, based on a linearization of the full Navier-Stokes equations around a mean flow.

\section{Appendix A. The Cummings-Elliott approximation}

In this appendix, we reproduce the argument of Cummings, as presented in Elliott [19]. We restrict to the situation of a pipe with no mean flow $(M=0)$, a situation where the analysis can be simplified in a substantial way. 
By considering a control volume corresponding to the interior part of the pipe (Fig. 1), the Euler equations admit a quasi-1D form

$$
\begin{gathered}
\rho_{0}^{*} \frac{\partial\left\langle u^{\prime}\right\rangle}{\partial t^{*}}=-\frac{\partial p^{\prime}}{\partial x^{*}}, \\
\frac{1}{c_{0}^{* 2}} \frac{\partial p^{\prime}}{\partial t^{*}}+\rho_{0}^{*} \frac{\partial\left\langle u^{\prime}\right\rangle}{\partial x^{*}}=-\rho_{0}^{*} \frac{P^{*}}{A^{*}}\left\langle v^{\prime}\right\rangle,
\end{gathered}
$$

where $p^{\prime}$ is the pressure, $\left\langle u^{\prime}\right\rangle$ the axial velocity (both space-averaged in the lateral direction), $\left\langle v^{\prime}\right\rangle$ is the average lateral velocity at the outer boundary of the inner domain $\left(r=R^{*}\right)$, and $P^{*}=2 \pi R^{*}$ and $A=\pi R^{* 2}$ denote respectively the perimeter and the area.

Combining Eqs. (A.1) and (A.2) we arrive to

$$
\left[\frac{1}{c_{0}^{*} 2} \frac{\partial^{2}}{\partial t^{* 2}}-\frac{\partial^{2}}{\partial x^{* 2}}\right] p^{\prime}=-\rho_{0}^{*} \frac{P^{*}}{A^{*}} \frac{\partial}{\partial t^{*}}\left\langle v^{\prime}\right\rangle .
$$

Assuming a wave-like perturbation proportional to $\exp \left(\mathrm{i} \omega^{*}\left(x^{*} / c_{\mathrm{eff}}^{*}-t^{*}\right)\right)$ and introducing the admittance defined by

$$
\langle\beta\rangle=\rho_{0}^{*} c_{0}^{*} \frac{p^{\prime}}{\left\langle v^{\prime}\right\rangle}
$$

One leads to ${ }^{2}$

$$
\left(\frac{c_{0}^{*}}{c_{\mathrm{eff}}^{*}}\right)^{2}=1+\frac{i P^{*} c_{0}^{*}\langle\beta\rangle}{A^{*} \omega^{*}}
$$

(equivalent to Eq. (46) in [19])

Using the admittance modeling (Eqs. (51)-(53) in [19])

$$
\langle\beta\rangle=\frac{i \omega^{*}}{c_{0}^{*}} \frac{L_{c}^{*}}{P_{c}^{*}} \frac{V^{*}}{S^{*}}
$$

one leads to the final result.

\section{References}

[1] B. Rajavel, M.G. Prasad, Acoustics of corrugated pipes: a review, Applied Mechanics Review 65 (5) (2013) 050801, http://dx.doi.org/10.1115/1.4025302. (24 pages).

[2] G. Nakiboğlu, O. Rudenko, A. Hirschberg, Aeroacoustics of the swinging corrugated tube: voice of the dragon, The Journal of the Acoustical Society of America 131 (1) (2012) 749-765, http://dx.doi.org/10.1121/1.3651245.

[3] Y. Nakamura, N. Fukamachi, Sound generation in corrugated tubes, Fluid Dynamics Research 7 (1991) 255-261.

[4] D. Tonon, B.J.T. Landry, S.P.C. Belfroid, J.F. H Willems, G.C.J. Hofmans, A. Hirschberg, Whistling of a pipe system with multiple side branches: comparison with corrugated pipes, The Journal of Sound and Vibration 329 (2010) 1007-1024.

[5] G. Nakiboğlu, S.P.C. Belfroid, J. Golliard, A. Hirschberg, On the whistling of corrugated pipes: effect of pipe length and flow profile, Journal of Fluid Mechanics 672 (2011) 78-108, http://dx.doi.org/10.1017/S0022112010005884.

[6] J.E. Rossiter, Wind-tunnel experiments on the flow over rectangular cavities at subsonic and transonic speeds, Aeronautical Research Council Reports and Memoranda 3438 (1964).

[7] M.A. Isakovitch, Scattering of sound waves on small irregularities in a wave guide, Akusticheskii Zhurnal 3 (1957) 37-45.

[8] J.S. Samuels, On propagation waves in slightly rough ducts, Journal of the Acoustical Society of America 31 (1959) 319-325.

[9] R.F. Salant, Acoustic propagation in waveguides with sinusoidal walls, Journal of the Acoustical Society of America 53 (1973) 504-507.

[10] A.H. Nayfeh, Sound waves in two-dimensional ducts with sinusoidal walls, Journal of the Acoustical Society of America 56 (1974) $768-770$.

[11] A.H. Nayfeh, O.A. Kandil, Propagation of waves in cylindrical hard-walled ducts with generally weak undulations, AIAA Journal 16 (10) (1978) $1041-1045$.

[12] A.M. Nusayr, Propagation of waves in rectangular ducts with sinusoidal undulations, Journal of the Acoustical Society of America 67 (1980) $1472-1476$.

[13] V. Pagneux, N. Amir, J. Kergomard, A study of wave propagation in varying cross-section waveguides by modal decomposition. Part I: theory and validation, Journal of the Acoustical Society of America 100 (1996) 2034-2048.

[14] P.M. Morse, H. Feshbach, Methods of Theoretical Physics, McGraw-Hill Science/Engineering/Math, New York, 1953, $2034-2048$.

[15] A. Maurel, J.F. Mercier, S. Felix, Propagation in waveguides with varying cross section and curvature: a new light on the role of supplementary modes in multi-modal methods, Proceedings of the Royal Society A 470 (2014) 20130743.

[16] A. Maurel, J.F. Mercier, S. Felix, Wave propagation in a waveguide containing restrictions with circular arc shape, Journal of the Acoustical Society of America 137 (2015) 1274-1281.

[17] A. Bostrom, Acoustic waves in a cylindrical duct with periodically varying cross section, Wave Motion 5 (1983) 59-67.

[18] L. Lundqvist, A. Bostrom, Acoustic waves in a cylindrical duct with infinite, half-infinite or finite wall corrugations, Journal of Sound and Vibration 112 (1) (1987) 111-124.

[19] J.W. Elliott, Corrugated Pipe Flow: Lecture Notes on the Mathematics of Acoustics, Imperial College Press, London, 2005.

[20] C.J. Nederveen, Acoustical Aspects of Woodwind Instruments, PhD Thesis, Knuf, Amsterdam, 1969.

[21] C.J. Nederveen, Acoustical Aspects of Woodwind Instruments, PhD Thesis, Revise Edition, Northern Illinois University Press, DeKalb, 1998.

\footnotetext{
${ }^{2}$ Note that in the notation of Elliott, $k^{*}=c_{0}^{*} / c_{\mathrm{eff}}^{*}$ and $\kappa^{*}=\omega^{*} / c_{0}^{*}$.
} 